\title{
ANI BC DDHNTES HISTORICOS
}

Ya en los años que precedieron a la fundación definitive de Buenos Aires, se sabía a oiencia cierta en la Asunción de I paraguay, que grandes tropas de baguales pastaban ${ }^{\gamma}$ las pampas a rentinas.

Cuando se pregonó en dicha ciudad la empresa de tal fundación, se ofreció pera el en ganche de soldados, como aliciente prinoipa 1, Ja apropiaoión de ese vagabundo ganado cabellax, tan codioiado a.1 pareoer por los pobladores paraguayos(1).

A1 efectuaxse, años despues de le citada fundación, la prom banza respectiva, dijo el testigo liguel Gómez:" .. que vio como por mandeto de dicho General Juan de Garay se apregonó.... que en nombre del Rey nuestro señor hacla merced a los pobladores y vecinos de esta Giudad del dicho ganado silvestre para ellos y sus sucesores...".

Ia pregunta se refería olaramente a ganado caballar.

Dijo el testigo Gaspar de quevedo:"...entiende que eI prinoipal intento-(de Garay)-despues de servix a Dios y a su Magestad fué, el de prometerles los dichos caballos y que sabe que sin los dichos cabalios no era posible sustentarse".

Para que el General Juan de Garay haya tomado como a lioiente prinoipe.1, para el en ganohe de volurtarios en la Asunoión, la apropiación de tal ganado wilvestre, debió tener la certeza de su existenola y de su gan abundanoia.

No podía pretenderse sacar los mestizos paraguayos de su tex cómoda y féoil vida en el "paraiso de Mahomel", sino ofreciéndoles pa sibilidades de un répido enriquecimiento en Ias inhóspitas riberas rioplaténses, donde sus padres habían sido tan oruelmente tratados por los indigenas y por la falta de alimentos.

Precisemente a ellos, habitantes de ese emporio de abundante y rioe dimentación, que era el puraguà, donde los espernoles y mestizos fretemizaban tan admimabiemente con los indigenas, no se los podía enตูอกี่อ.

Es bien sabico que el prooeso de una fundación de nuever oiludeconen esa época, tenta quo ostar preoecido do una buena explosactón del texrono y una merosa truititación ante la corte, para a securaz dereoho 
y legitimarlos, previo estudio de los medios de ejecución.

Esta es la razón por la cual pu.ede asegurarse que, desde tarios años antes del aro 1580 en que se fundó la 2a.Buenos Aires, ya. se sabía en Asunoión del paraguay que en las pompas veoinas a las riberas rioplafftenses abundaban los tan oodio iados baguales.

Esta información histórica resulta muy útil para interpretar el mapa titulado "Tab ladel gran rio", oonfecoionado por Sebastian Gaboto a.I pareoer eñ el ano 1533, donde aparece un cabal10 sa.lvaje on la Ilanura que se extiende entre las siemras y el rio parané, figurandovun tigre en dicha sierra, dibujos correotamente hechos y de mucha vita

Poco tímpo despues de fundarse la ciudad de córdoba, suco-

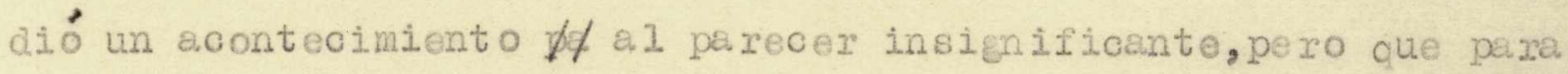
Los que investigamos historia, nos resulta aotua Imente trasondenta I: el falleoimiento del ind'o Gonzalo, que en una carretela tirada por una "yegua hoberall venía desde el Rio de Navidad, hoy Rio 2•, a Ia ciudad de córdoba.

Intervinieron de inmediato las autoridades para efeotuar el respeotivo inventario, porque el tal Gonzalo al parecer no ten ía herederos.

"Joen de No lina Nabarrete a loble hordinario desta oiudad de córdoba e sus terminos e juridicion e juez de bienes de difúntos por la presente yo vos mando a vos Luys de Abreu de Albomoz a Iguadil mayor desta dicha ciudad queluego como os fuere notificado este mi mandariento vays con el presente esoribano a l, pueb lo de omarasacate... donde residia Gonzalo indio natural de los reynos do ohile, ya difunto $y$ en virtud deste mi nombramiento hazed inventario de todos los bienes que dexo por su fin y muerte".

Esto sucodía en el año de 1584.

Lo que encontró en la pequeña carreta el inventariador, ya nos empieza a poner en presencia de algo insólito:entreotras muchas oosas ol indio Gonza Io viajaba oon un aroabuz, una lanza de fresno o on su hierro, dos frenos jinetes-(tipo árabo)-oon sus riendas, un par de espuelas pico de gurrion, una silla gineta on sus estriberas de 
hiemo y garruzeles y cinoha con su coraza de tigre, etc?'

cono vemos, un equipo casi completo de guemero de la época, a. 1a. usanza árabe.

Nuestra admiraioión se a g granta a 1 enteramos que en Una rea sacate, ribera Norte del actual Rio $2 \bullet$, este indio ohileno era propieta= rio de una Estanguela, donde tenía una majada de ovojas, otra de cabras, una tropilia de llamas y un pequeño haras en que oriaba caballos para vender a los españoles de córdoba.

Dicho pequeño plentel equino se componf́a de oinco yeguas $y$ "un potro castaño tresalibo con una estrella en la frente". Tres orf́as en el corral completaban este renglón.

pero en la chacra de Diego de Loria en la oiudad, tenf́a e I indio Gonzalo "dos potros comados"。

Y en un oofre que llevaba en la carretela, entre otros papeles se enoontróllun oonocimiento de Juan de litre de quarenta pesos por dos rosines que parece le vendió el dicho difuntol. Es deoir, un documento segun el cual, dicho famoso capitan reoonoce haber reoibido los caballos, a pagar en el futuro.

Producida Io almoneda en el año 1586 , vemos 10 siguiente : "opbrado a Juan de Mitrepor los dos cabalios que debía a Indio GonzaLo diez varas de paño de Tucuman".

Resulta pues indudable que un indio ohileno-(de cuyo, talvez d de fendoza)-era indio libre y estenciero no lejos de la Ciudad de oórdoba, instalado desde que se fundó esta ciudad cong una estanzuela. en Is märgenes del Rí $2 \bullet$, en la cual oriaba potros que domaba y vendía a los fundadores. El extenso documento, que fué cerrado y archivado en el año 1588, figura como Exp.lo-del Logajo 2 de la Esox.12, del Arohivo Histórico de córdoba.

Un indio ohileno(ouyano)oriador y domador de potros en tal. época, presentado como preoedente histórioo tan formal, debe sex tenido muy en cuenta al estudiax el tema de las relaciomos existentes entroindios y cabalios, en la época anterior a la fundaoión definitiva de la Oiudad de Buonos Airese

El mismo Achivo Histórico de córdoba nos proporoiana otros documentos muy informetivos sobre la re leciónllindio-ca a all de nups. 
tras pampas, en esa primera époea.

Asi venos en el Exp.5-Leg.24-Esor.1a.-año 1611, la demanda interpuesta ante la Justicia de córdoba por una veoina de Buenos Aires, contra Alonso Diaz Caballe ro vecino de ó́rdoba, por haberle maloqueado los indios daguanen del rio Gurauca, hoy rio de los Arreoties y deI salto(B...)

Resulta que estos indios fueron encomendados al capitan R fael Despindola, vecino de Buenos Aires, por el Tte, de Gobemador Rodiso Ortiz de zérate.

Por fallecimiento de ese primer Bnomendero, el Gobemador Homando de Záráte eno omendó esos indios en el año 1594 al veoino de Buenos Aires Alonso pa lomino, ouya viuda dona Maria del dastizlo, fué la que demandó años despue a 1 ma loqueador Diaz Caballero.

Resulta aquí oportuno plantear el interrogante de quien y ouando se interesó por enseñar a los indios caguanon a pillax y domax potros cimarrones.

Ia distanola del rio Gurauca a Buenos Aires, el género de vida en esa primera épooa y la suerra de ingios que ya estaba planteada desde la $1 a$. Buenos Aires, hos hace pensar que con esta Enconienda de indios debió pasar lo mismo que pasó con muchas de ó́roba y del Tucuman, que resultaron simplemente nominales y al solo objeto de obtener título delveoinollue traía aparejado el de hijoda.Igo:

Asi vemos que palomino que recibió la moomienda en 1594, reoien tomó posesión de ellà en el año $1602^{\prime \prime}$ en la plaza publica de la oivdad de la Trinidad....ante el Aloalde ordinario dapitan Biteoreasco de Mendoza....en la persona de Minchaba. I hermano del cacique seca Idavan ... de (t1 nacion caguanen...." .-

Entre los indios comechingones el subfijo BN simifioaba pueblo, equivalente a los que los pempas llemeban naoion.Oreemos por lo tanto que esta tribu del rio Gurauca pudo ser de apellido caguan erz si fueramos a aplicax la libertad interpretativa que utilizó Monseñox pab lo cabrea en estos problemas linguistioos, podrfamos llegar a le o onclusión de que el nombre de la tribu debió ser Cagua I, que exa la pa labra que tenfan los pampas para designar al cabalilo. 
de baguales de estos indios del rio Gurauca, es porque a. I maloquearlos el Capitan Diaz caballero en el año 1611, 11evándoselos a la Roducoión que tenia sobre el Rio $3^{\circ}$, de la jurisdicoión de córdoba, ollos eran ya eximios domadores de los bravios bagua les pampas.

Asi 10 comprobamos ox el Exp.2, Leg.53-EScr. $1 a$, deI oitado Arohibo Histórico, donde ellos informaron a I Juez Visitador:" que alsunos dellos han salidio alsunas vezes a coxer yeguas cimaxronas y potros para su. Enoomendero y ellos doman potros de los que coxen y su. Mnoomendero les a repartido cordellate y sayal y sombreros y camisetas $y$. Ies haze buen tratamiento".

"ت1 dicho teniente de Gobemador (de ósrdoba) dixo que por ser reoien reduzidos estos indios y ser pampas por agora los dexa en el estado que los a.1lare....que si los dichos indios de su volunted. no quisieren trabaxar no les apremie a ello y queriendo les pague su. trabaxo...".-

conooiendo, o omo conocemos al detalle, las actividades guexrexas de l capitan Alonso Diaz caballero y las otras Pnoomiendas de indios que tenia en las sierras de córdoba, podemos asegurar que no tuvo tiempo para convertir a los caguanen en domadores de potros oimarrones. Queda en:pie la pregunta de si tal enseñanza fué heoha por doña Maria del castillo, o por su marido pa lomeque, que tomó posesión de la moomienda ocho años despues de haberla reoibido.

Con estos antecedentes liegamos aia conolusión de la bundanota de caballadas oimarronas, desde las llanuras de senta Je, hasta la costa at léntica y el Rio 3* de córdoba, lo oual se complomenta con informeciones históricas que "veremos mas adelante, de que 11egaban hasta el extremo sur de la Patagonia.

Y correlativamente con tal abundancia de baguales, comprobamos la habilidad de domadores de potros por'parte de los ingdios, on Ia época de la fundación de córdoba y de la 2a.Buenos Aires.

Como un complemento, aunque no indíspensable de esta primera conclusión, me parece interesante copiar algunos péracafos del excelente trabajo deI Dr.Agustin zapata Gollan, sobre el caballo de la conquista(2). Dioen asi: 


\section{$-6-$}

"En el paraguay ya se tenian notioias de la existencia de las tropilitas alzadais en las pampas del Rio de la Plata, ouando Garay se disponía a organizar la expedioión con que fundarf́a de nuevo a Buenos Aires,y fué ese,el, prinoipal alioiente de los hombes que le acompanaron en la empresa".-

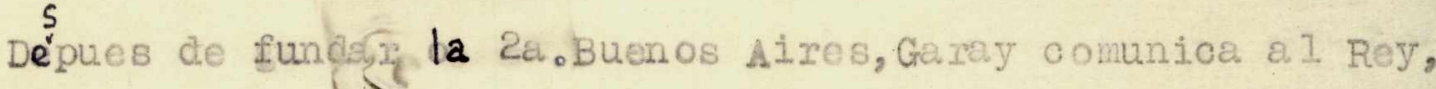
que estos baguales han sidp vistos solamente desdelejos"lhasta agora, por ser la tierra tan rraza y llana no emos podido tomar ninguno ni emos tenido posibilidad ni espacio para hacer corrales que son menester hazerse grandes en las aguadas".

claro está que en esta primera época los mestizos paraguayos

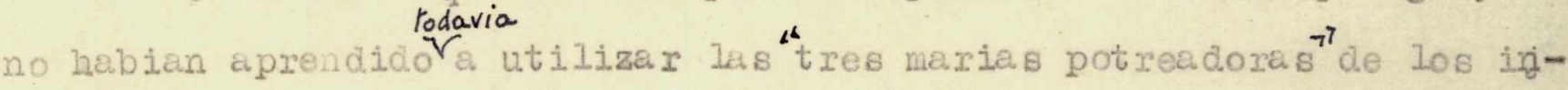
dios pampas.

El 7 sorero de la nueva oiudad, Hemando de Montalvo, informa en el. año 1587 que l ganado caballar cimaxun"pasa de cien mil cavesas arriva estan dentro detreinta le guas de este puerto aunque se torien oon trabajo y peligro de yndios que estan como el primer dia que a.qui se vino a poblax".

En esta referenoia de las treinta leguas, está el rio Gurauca de que ya he os hablado, justamente en tal limite. Ia información de que los indios estan en oompleta rebelaía en esa feoha, oertifica la hipótesis que hemos sustentado de que no fueron los fundadores de Buenos Aires quienes enseñan a domar potros a los indios caguanen.

De otro interesante libro, del oual me oouparé mucho en este estudio(3) tomamos la informa oión de que en ell año 1535 ouando los Querandies mataron a.I Capitan Diego de Mendoza y otros eepañoles en eI combate de 1 rio de la Matanza (1afundación de Buenos Aires)a los espanoles derrotados que huyeron"de a caballo" los hubieron muerto tambien, sino hubiera sido por la intêrvenoión de la infanterf́l"por ser oomo eran los indios tan lijeros y tan diestros on atar los caballos o cn bolas que traf́an". Segun una carta xidraxtax escrita por el testigo prosencial Pranciscô Vilila Ita.

Por postexior documentaoión se ha comprobado que las querandíes, disponían de estas famosas"tres marias potreadoras", distintas $y$ 
mas pesadas que las boleadoras guanaquueras y avestrucems. A.gun indioio se vislumbra aqui de que no inventaron este admirable instrumento de ea mayor, para aplioarlo oon los oabalilos a - Buewar Sires $\rightarrow$ de la $1 \underline{a}$ suenos Aires, sino mucho antes.

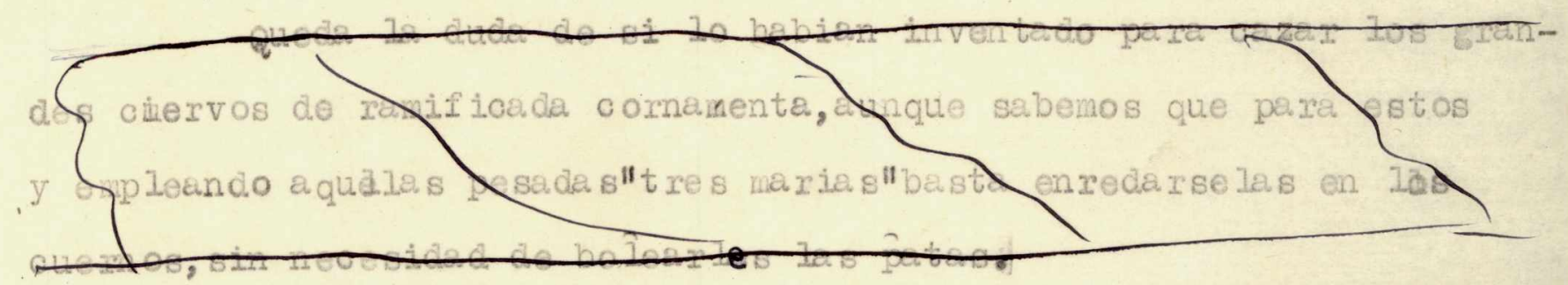

otra oita que enontramos en este libro(3)nos informa de que" Satriento, mandado en 1579 del Calla en busca de Drake, en el estrêcho de Magalianes, vió a los indios oazando montados en caballos y haciendo uso de boleadoras". Se refiere esta información a. famoso Capitan de la o onquista sarmiento de Gamboa.

Será necesario refutar documentadamente estas informaoiones históricas, para que pue da negarse la conolusión de que,al comenzar el último cuarto deI siglo XVI, los indios estaban en dominio del caballo y eran capares de a poderarse de p̈tros oimarrones en plena pampa y de domarlos a entera satisfacoión de los capitanes españoLes y esto suoedía desde Santa Fé hasta el Estreoho de Maga lilanes y desde el litora I At lántico hasta la cordillera. 


\section{IAS GRANDES BAGUA IADAS DEL SIGIO XVI}

Si hacia el año. 1575 vagaban por nuestras pampas las grandes tropas de baguales, que la documentación histórioa se ha cncargado de hacemos conocer, se impone a los investigadores la tarea, no por mora curiosidad, sino oon un ooncepto oientífico, de aolarar su prooedencia.

Fn las mismas pampas vivían aunque no con i qua I abundancia, las trópilitas de ciervos, suanaoos y avestruoes, que compartian on los baguales los altos pastiza les pampeanos, matizados de manchones de axbustos, cardales y pa jonales, on los ouales disimulaben su presenoia los senguinarios tigres y pumas,que vivian gordos y felices en ese paraiso de came fresoa, mu Itiplioando sus crías.

Y allí estabo tambien presente el fomido y agil indio pampa, disputańdoles las aguadas y las presas. Sus amas,no improvisadas, sino evolucionadas a traves de los siglos, fueron, ademas de la feoha y piedras arrojadizas, los tres tipos de bovadoras avestruceras, cuanaqubras y potseadoras. Esta líltimas, muoho mas pesada, constituyeron las que se cieron famosa"t"tres marias".

El indio, los oiervos en sus tres distintas especies, el aveztruz y los lamas en sus cuatro especies distintas, venian desde muy lebios en el tiempo:desde el pleistooeno.

pero desde oúando los baguales paseaban su soberbia estampa por nuestras ubérrimas pampas?.

Io pa Ieon to lo f́a nos prueba que al final del sus oompañeros pampeanos, queitambien habitaron en nuestras montañas.

Todos ellos pasaron la terrible prueba biológica de las arondes y prolongadas liuvias de oenizas voló́nicas"aoidas de 1 final 1 Pleistoceno, de ellos hace unos 10.000 años y se adentraron profundamente en el Holoceno, en omparia del gigantesoo liylodon, de jando entre las blanoas cenizas que envenenaron los pastos, los esqueletors de la sigantesca fauna pempeana, en la cual se destacaban esos enormes quirquinchos de la fomilia de los Glyptodontesfft, que pesaban mas de una tone leda, rega lo pantagruélico de indios y tigrese

De este problema paleontológioo nos oouparemos en el próximo capítulo. Pero es ocnveniente antioíra. 
periodo seolósioo Ilamado Reoiente u. Holoceno, se o omprueba la existenoia de nuestro oaballo autóotobo, conocido científicamente con el nombre de Equus Reotidens, que en nada diferé́a de nuestro caba 110 oriollo del siglo XVI.

Para comprobario están sus huosos en nuestros museos. Sin ombargo,nuestra cienoia aotua I niega que el cabal10 oriollo deI siglo XVI desoienda deI Equus Reotidens y oomo argumento Insiste en que la Arqueología no lo ha probedo. Volveremos sobre e I

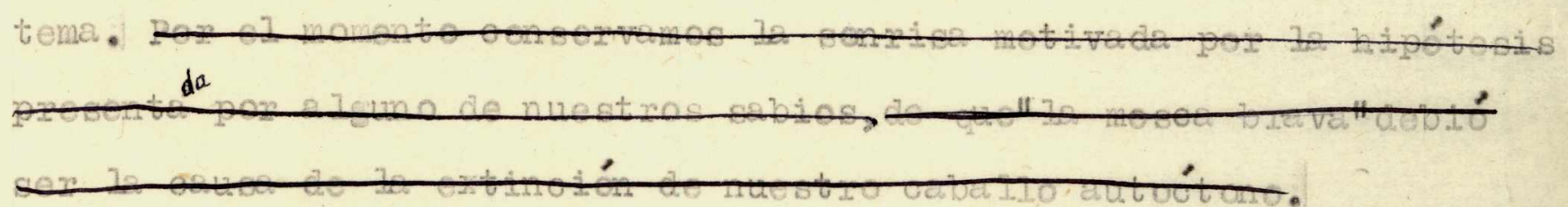

Para explicar la existencia en el último cuarto del siglo XVI, de tan ren cantidad de caballos on nuestras pampas, los historiadores se han valido del argumento de que ellos descendín de los oinco yeguas que quedaron, a mediados de dicho siglo, al ser destruída por

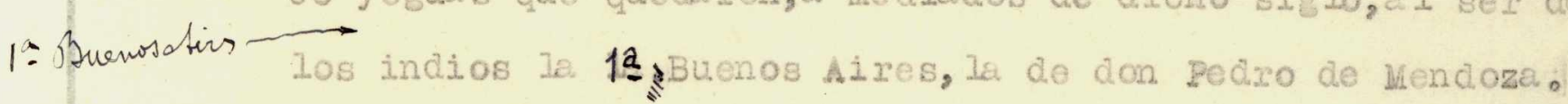

Haremos nuestro propio plenteamiento de probabilidados, haoiendo interventro los factores reales del problema, pero es necesario y justioiorio dotamemos aquí pera ocasiderar e lexo lente trabajo sobre ol tema del investigador Anibal cardoso, fochado en el año 1912 (3).

Empezaremos por transoribir el pármafo del autor della Argentinall Ruy Diaz de Guzman, escrito por este historiador de la oonquista, en el año 1612 , on el cual empieza el estudio col Sr.oamdoso. Dice asI:

".... que este puerto fue pobledo antiguamente por los oonquistadores-(se refiere a la 1a.Buenos Aires)-y por oausas forzosas que se ofrecieron vinieron a despoblerie, donde parece que dejarom cinco yeguas y siete caballos, los ouales al día de hoy han venido a tanto multiplioo, en menos de 60 sanos, que no se puede numerax, porque son tantos los caballos y yeguas que pereocn grandes montanas, y tienen ocupados desde el. Cabo Blanco hasta el Juerte Gaboto, que son mas de 80 leguas, $\mathrm{y}$ llegan adentro hasta le cordiliero.l.

En varias péfinas discute cardoso la informaoión sobre las oinoo yeguas de Mendoza, terminando por negar suè existenoia, insis- 
tiendo posteriormente con las siguientes paleinas:

"Ya he demostrado anteriormente que no quedaron en Buenos Aires oaballos abandonados, pues los comieron los onquistadores acosados por el hambre horrible que padecieron durante el sitiol.

Y agrega una informaoión deI año 1581:"Relación de las Provinotas del Rio de la Platal, que un español se comió a su propio hex'mano que había muertamomplètando el ouadro oon esta observación:"tam-

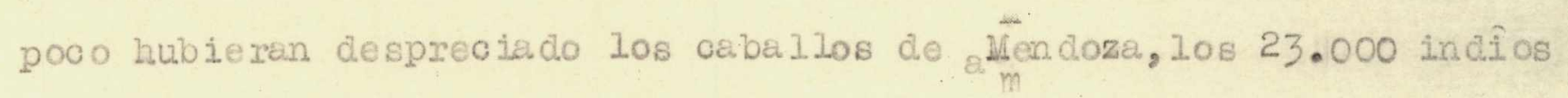
stiadores".

\$1 cáloulo probable de reproduooín de las oinoo yeguas suponiendo que hubieron existido, to hace caxdoso sobre la base de tan solamente un quinto de pérdida de. Jas orías y aoeptando que las potrancas pariesen a los tres años de edado

Planteamiento optimista de 1 problema,con el oual 1 deduce que, entre los años 1537 y 1580 la brillante reproducción de este oriadero pampeano, presentaría un oojunto de unos 1580 yeguarizos"de todas edades".

Hò co todaviâ otro oáloulo mas oplimista, casi fabuloso, o cm un resultado de unos 23.000 yeguarizos, oomentando:" Esta sum esté muy lobos de cubrir la costa del rio de la plata desde el Fuerte de Gaboto hasta el Cabo Blanco ... J ni siquiera aloanza a la oifra de 80.000 a bezas en que oáloulob esa hacienda el Tesorero yontalvo en I581".

Por mi parte aprecio que todos estos cáloulos son inútiles y el resultado sería el mismo con cinco yeguas, que oon el doble o triple pues los factores que han debido intervenir para dificultar la reproducción de estos animales, son los mismos que para los ciervos y suanacos, que nunca pudieron pasar de pequeñas tropilias, teniendo igual gestación.

Los indos y los grandes camívoros de la pampa fueron la oausa de esa reproducoión restringida y los que motivaron ese vida de terror en lis velooes tropilias de ciervos $\mathrm{y}$ suenacos, que hacian ton inquieta vida en la pampa.

Lo 'mismo' hubiera sucedido con los desoendientes de las oinco yeguas de kendoza y no debemos olvidar que el plazo de lal gigantesca" 
reproducoión sodo corrió desde el año 1535 a.1 1580, o sea, una duración de $\tan$ solamente 45 años.

Ins grandos bagua ladas que asombraran a los hombres $\mid$ de Garay, sometidas a los mismos elementos de destruoción que asolaban a ciervos y guanacos, Ilegaron a ser tan srandes porque ya entraron con abundan oía al Holoceno, 0 sea, 10.000 años antes de la conquista española,

In otra forma es absolutamente inexplioable que, en tan solamente 45 años, la descendenoia de oinco yeguas pudiera contarse por centenares de miles pues si en el seotor vecino al 2 - Buenos Aires, fueron apreciados en 80.000 , oa loulemos los que habría en todas nuestras Ilanuras, desde el Chaco hasta el Estreoho de lagallenes y desde la oosta At lántica hasta la cordiliera.

He confeocionado.

rouadro Faplicando probabilidades exageradamente optimistas, con el objeto de determinar un número quie pueda ser comparado o on los proporoionados por la información histórica.

$$
\text { Si bien es oierto quevtona/ia parioión }
$$

de 2 en 2 años en realidad habría yeguas que lo haxían a veoes en años seguidos, en en cambio no tengo en cuenta la acción do los oazadores querandíes, ní Lampoco la acoión de tigres, pumas y el gusano on las heridas.

Los indios pampas siempre fueron amantes de la carne de potranoa y esto ocnstituye un elemento de juioio muy valioso en este ca. so. Tambien apreolaban en forma sobresaliente el ouero de los equinos, tanto para sus chozas, como para otros muy variados usos.

Estas solas consideraciones bastan pera llegar a la conolusión de que, si se tomara en ouenta la acoión de los caradores hubiera sidor per perduración de los equinos en muy pequeños grupos y solamente conside rando milenios, podxíamos 1 Ie gar a las grandes tropas de ba gua les"muy ariscos" que vieron los hombres de Ga. ra. $_{0}-$

ese

Para confecoionar «" cuadro he towado los siguientes datos: parioión cada dos años, las potrancas paren a l oumplir los tres años, durantela prición se pierden ei $10 \%$ de las oxías,ninguna otra potranoa 
se pierde,todas las potrancas son fértiles; de las yeguas madres se piecle por muerte, inutilidad o vejez el $5 \%$ anual, ner que paren durante 20 años.

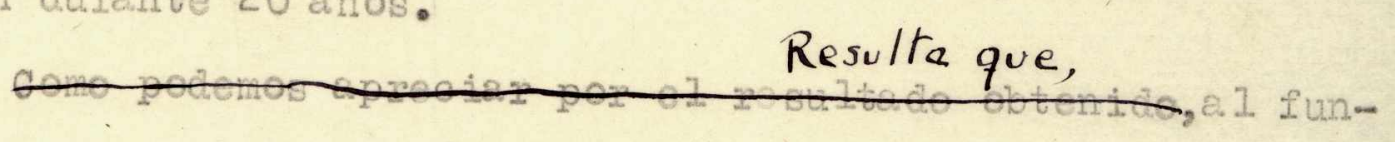

darse la 2a.Buenos Aires, vivirían muy azarosamente en nuectras pem-
y llanuras, pas) Yen una extensión superior al millón de kilómetros ouadrados, una cantidad de ye guarizos muyminferior a 10.000.-

De esta cantidad repartida on pequeñas tropillas,alsunas de ellas pudieron ser vistas por los hombres de Garey, si se hubiesen mantétido ais ladas de las grandes bagua ladas autótonas. Pero 10 mas probable es que, desde el prinoipio si pudieron sobrevivir, se hubiesen mezo lado óon esos con géneres.

Pero estoy en un todo de acuerdo oon dardoso (3): esos poool abalilos de la 1a.Buenos Aires,no pudieron escapar a los miles de querandíes que tan mal trataron a los hombres de pedro de Mendoza.

In el ouadro adjunto puede verse que las 5 jeguas de Mendoza,no tuvieron pérdifa alguna,ni en su numero ni en el de sus oxías, durante los dos primeros ar̃os.

En el teroer año de andar escapando a los cazadores - hombres y fieras- reoien pierden una potranca.

A. año giguiente, se pierde una yegua madre, por vejer, muerte o inutilidad.

En el 5- año no hay perdida alguna, ni en las potranioas

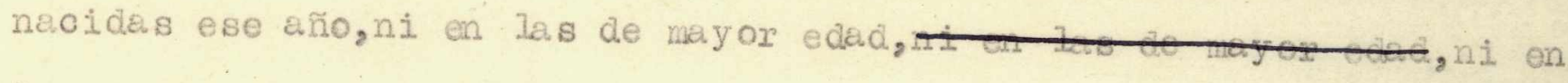
Las yeguas.

Tal es el ritmo del ouadro. Si en los años siguientes aumen tan las pérdidas, es por el mayor número de yeguarizos, pero he mantenido el ritmo del 10\% de pérdidas para las potrancas del año y $5 \%$ para las Jeguas madres, todo ello anua. Imente.

Tomaremos ahorat un notable quista española, oon oriaderos de caballos bien ouidados y bien protegidos.

Se trata de la époda en que Hemando de Soto onquistó Ia I1orida, que es la misme 


\section{(2) -13-}

Aires. Ia informa ión la temő de la famosa obra sobre los caballos de la conquista, de ounninghame Graham (4).

roda la información, que es muoha dada por este autor, prueba la penuria do los onquistadores por oonseguir caballose

Pese a la marcada protecoión del Buperador carlos $V_{2}$ Soto no oonsiguió mas de oien caballos en España para su gran empresa. Pero al llegar a cuba se encontró oon que allf́ existín vordaderos oriaderos desde el primè desemaroo español, habianse multiplioado enormemente. Los alií residentes criabăn los, en especial, para venderlos a los conquistadores que iban a las campanas del perú y de Héjiool.

Soto "se asooió allí a un rioachollal cual nombró enseguida "teniente señera. I del ejeraito y de la fiotallintrodujo como oapitä̀, oincuenta cabalios y una hueste de indios y de neeros,amen de treinta y séis caballos mas pera su silita y séquito personall".

Vale decir que, la contribución que el ricacho nombrado 2- Jefe del Éercito y la rlota, aportó en oamio de tan insigne honor, fué solamente de 50 caba. Igaduras pera el Ejeroito. Se sobreentiende que se trataba de cabalids y yeguas.

Tal fué la cantidad que pudieron proporoionar los famosos oriaderos de cuba, tan prósperos en esos 35 años de su estableoimiento. Para juzgar este asunto debe tenerse en ouenta que ningun soldado de caballexía en esa época, se conformeba o n tan solamenté el montado;exijían siempre un caballo de repuesto,por 10 menos.

Ia numerosa información recogida en el Archivo Histórico de córdoba, nos presenta ejemplos numerosos de tres caballos por jinete y no constituyen excepoión los casos de cinoo.

con el ejemplo presentado de los prósperos oriadoros de Cuba, podemos tener una idea de la reproducoión de yeguarizos,bajo vigilencia y protecoíón, partiendo de la base de buenos planteșles de yeguas. Podemos imaginamos el resultado a obtener, ocn oinco jeguas madres, abandonadas a su propia suerte en las inmensas pampas arcontinas, pobladas de tandios tan hábiles en el manejo de" las tres maxias" y tan ávidos de la came de potranca, a los evales debemos aere a ar 


\section{NUESTRO OABALIO AUT OCI OVO}

Estudiaremos a qui un muy interesante problema, que fué debatido con entusiasmo a principios del oorriente siglo y que ya ha sido dado por finiquitado, de acuerdo a la generalizada opinión, que oan tanta certeza como laoonismo, nos presenta el Sr.Justo P.Scenz (hijo) traduotor de la obre oitada anteriormente(4).

A propósito del caballo autóotobo de que nos habla qunnighame Graham, dioe el Sx. Saenz:"Ia teoría de que este anima l existió en Ins Americas antes de la llecada de los españoles esté completamente en desuso y nadie medianamente onterado del asunto se atrever hoy a sostenerlal.

Partimos de là base de que nadie niega la existenoia en nuestras pampas y montañas del caballo fósil, el cual 1 le gó hasta el comienzo del Holoceno en companía del resto de la fauna pampeana de grandes mamiferos.

Lo que se discute y se niega,es que las enormes bagua le das que se vieron en nuestras pampas en eI siglo XVI, fueran desaendientes de dicho oabal1o autóctono. Segun la opin ón generalizada esos oentenares de miles de baguales del siglo XVI, proceden única y exolusivamente de las oinoo yeguas de la 1a.Buenos Aires, a las cua Ies pudieron agregarse a Iguns otras yeguas escapadas de otros Reales, aunque no se nos preoisa de ouales y ouando.

Yo tambien participé, sin ningun análisis por mi parte, de aquelia opinión generalizada. I por oulipa de tal prejuioio he tirado huesos de caba.110 encontrados en los estratos 1 lamados pre-aimarenses, por considerarlos como provenientes de cabalios de la conquista.

Ha.ce tres años leí el libro de cardoso(3) y 10 puse de lo, menospreo fandolo tambien.

Pero mis recietces investigaciones sobre el Ho loceno $\mathrm{y}$ muy especialmente la comprobación de que entre los estratos pre-aimarenses, existe un estrato constituído por un "humus fósil", que en mi rooientefolleto llamollestrato negroll (5) me han convenoido de que este problema debe ser pueșto nuevamente en discusión, oantinuandose la abandonada investigación. 
El nuevo planteamiento es el siguiente sun hueso oncontrado en la tiemanogra no es neosariamente reoiente; si esa tierra negra pertenoe al humus fósil No 1, puede tener hasta $\mathbf{8 . 0 0 0}$ años de antigüodad NMd (resultado obtenido mediante el $C_{14}$ ).

El Equu reotidens pasó la barrerá del estrato o de la serie Doexing cotrespondiente a las grandes Iluvias de cenizas voloánicas d́cidas, haoe unos 10.000 anos y sobrevivió durante el primer teroio del. Holoceno, oorrespondiente a la deposioín de I I loess puivuru Iento amaxilio o laxo, que Ilamamos oordebense $(5)$.

En oulatiera de nuestros Museos de Cienoias Naturales existen huesos de Equus rectidens oorrespondientes a este periodo geológico. Yo mismo los tengo en mi pequerio museo de la Pampa do olacn. El Equus reotidens vivió no solamente en las pampas, sino tambien en las sormanfas.

Muestro gran investigador Ameghino era de aquella opinión y su compañero de investigaoiones de esa época, el Dx. Doeringe lo señalafth o laramente en su serie estratigréfioa $(6)$.

Pefo este líltimo investigador acepta que el Equus rootidens vivió hasta una época aun mas reoiente,al incluirlo en su estrato b' que o cresponde estrictamente al pre-aimarense.

Bnoima del estrato b' de Doexing no existe mas que el estrato a de tierra negra que han dado en Ilamarllaxianense", atribuyéndolo á "post-oonquista española".

$$
\text { Aquí reside preoisamentè toda Ia difioultad de una }
$$

interpretación correota, pues ese poderoso estrato superior de tierra negra o capa vegetal, no puede haborse formado en tan solamente 4 siglos.

Es un srave error que perjudica la investigación arqued lógioa, ol llamax AHIANENSE a la capa de tierra negra, dándole una antiö̈edad equiva lentéa la llegada de los espeñoles a América.

\$ si por efecto de la demudación, lo que se presenta on la superficié deI suelo es ellhumus fósill oorrespondiente al "optimum climátioo"(5) Jentonces la antigüedad del objeto arqueológioo puede 11 gar hasta los 8.000 años (información del $C_{14}-a n ̃ o 1956$ ). 
Basta esta consideración, pare ponemos en guardia ante oualquier desoubritiento de huesos de caballo en este estrato geoIógioo y para que tomemos la precaución de suardarlo, hasta que podamos determinar su. verdadera edad, mediante la aplioación de 1 mo-

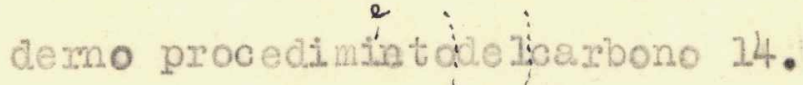

Este procedimiento aplicado a huesos que recojamos de ex-profeso y tambian alos que se conservan on nuestros yuseos,provenientes del Holoceno, nos permitifan resolver el problema de nuestro caba.110 autóotono.

Por de pronto,su aplicaoión reoiente, en huesos de cabalid oxtraÍos por Bird de la oueva de Palli Haike al Sur de chile, dieron porirextado una antigüedadide 8.500 años, Corresponden por 10 tanto a nuestro dordobenise, del cural tenemos muchos huesos de cabalio, ouya edad la hemose preolado entre 9.000 ₹ 7.000 años, sin neoesidad do reouritis al procedimiento deI c 14.

Lo que neaesitamos es apliarlo a los huesos extraidos. de la tierra negra, para saber si el caballo siguió viviendo a traves del "optimum olimétiooll en oompañ́a del oiervo y del guanaoo.

Y cabe aqui hacèr una observaoión muy importante: si el caballo autóctono fué cepaz de sobrevivir a la terrible prueba bioIócioa del pexiodo de las grandes lluvias de oenizas volónicas áoidas, que envenenxon los pastiza Ies, pasahdo despuešli por ol periodo de dos a tres mileniós del Cordobense, de olinga muy adverso para la vida de los mamiferos, el oual oulminóp con el pexiodo luvioso cuyos vesticios: Lo constituye el estrato bll de la serie Doering,nosotros debemosipreguntamos si es lógico suponer que dicho movedizo y resistente. máfero pudo extinguirse, preoisamente durante el periodo liamado "optimum olimático", en que tanto prosperaron los otros mamiferos que fueron sus compañeros durante los tres milontos precedentes, tan adversos desde el puthto de vista biolósico.

El caballo pudo extinguirso en nuestras pampas y montaRas durante eI seco y polvoriento a lima deI periodo

Cordobense, el oual oorresponde al estrato bu' do lo.

serie geológica de 1 Dr.Doerins, que se especializb en estas investiga- 
oicnes. Pero este mismo autor seña la la presenoia del Equus rectidens en su estrato bl que oorresponde al ya vaxjas veces citadolloptimum olimátiool.

, S Hstas consideraoiones nos oblican a abandonar la posición néativa on que homos estado colocados, all acoptar como definititiva la tesis sobreila extinoión total de nuestro caballo autóotono. Es indudablo Ut que en el porvenir solin encontrados huesos de oabalto on los terrenos humíferos que son desi mados como "pre-aimarense".

He sta tento puedan ser ana lizados por el prooedimiento de1. o 14, habrá espeotalistas que tratarén de aplioar el método paleon o to lógico para sus s c la gifioación. I a qui se presentarán nuevamente difioultades debido a los materiales de comparaotón.

Ia diferenoidotion debe hacerse o omparando oon huesos de ceballo ouya prooedencia europea sea indudable, pues muy bien puede suocder quie ol naterial de o omparación conservado en los Museos corresponda a. Io que llamamos"caba 110 oriolio" desoendiente de los bagualee de I siGlo XVI, ouya asoeñdenota es precisamente la que osté en discusía.

En el extenso malisis que hace dardoso(3)en su Capitulo III, titu Jado TESTIMATIOS: especies fósiles del cabalio, vanteriores al Equus reotidens. No oreomos que sea neoesaio extender tanto el estudio, pues el problema en disousión es muoho mas simple y conoreto, pudiendo resumirselo asi: los huesos de oabal10 que se enoutgran on la tierra negre de nuestro territorio, 00mesponden a. caballo europ 0 actual o a nuestro Equus reotidens? De I Gap Ítulo oitadoide Cardoso (3) tomamos los siguientes pámpatos:

"... I Dx.Ameghino habia enocontrado enlos "paraderos" indios de canada de Rocha on Iujan, restos del Equus rectidens con señales inequivocas de quelos quezandíes se alimentaban de estos animales...". "Compar n do la oabeza de Equus réotidens- (un oraneo o an servado on este Museo)-con la de cabailo criollo que posee este Museo, se nota. la somojanza de 1 perfil y el abovedado de la frente que xiste entre ambos". "Este ca jéo ter, Jumadoloabeza d.camemeda" es un lazo de unión 
entre las dos especies, demostrando una vez mas 01 abolengo netan te americano del caballo oriollo, pues el oaballo europeo tiene el. perfil reoto, la frente plana y la cabeza pooo voluminosal.

Stguen sus observaciones releolonades ocm otras carecta risticas del craneo, de las vertebras, de las extremidades, oto.de aouerdo a las cuales elloaballo oxiollo" se asemejaría mucho mas al. Equis reotidens, queal cabalilo europeo.?

No. debemos olvidar que este folleto de carcioso fué publiad do en el año 1912 por el Museo de Otiencias Naturales de Buenos Airath? del ourl habia sido Director en épooa reciente el Dr.Ameghino.., ar y del oul I erajefe de la Secotón de paleontología su hermano darlos Ameghino.

- cerraremos este capítulo con el primer párrafo del dapitulo IV, que cardoso dedica a "testimonios Arqueológicos e Históxioos". Dioe asi:

"Los testimonios arqueológicosu serían in'umerabies, si a I establecer la edad de losiobjetos hallados, no se hubiera partido de una base erronea: los caballos de Lendoza. De esto resulta que al haoor un hallazgo arqueolósico, por mas antiguo que sea, en cuanto apareoe un hueso de caba11o, un dibujo, o un objeto oualiquiera que indique este animal, se olasifica el hallazgo cono posterior a la oonquista del kio de la Plata".- 